\title{
Oral snuff
}

Addiction Ontology

\section{Source}

Addiction Ontology

Definition: An oral tobacco-containing product that is composed mainly or exclusively of moist, ground or powdered tobacco that is processed to make it suitable for use by a person placing it in the mouth between the gum and the cheek.

Comment: This class includes snus as well as other oral ground or powdered tobacco products that are not chewed.

Curator note: People often confuse oral snuff with snus and it is important to recognise that snus is a subclass of oral snuff and not the same as it.

This definition was imported from the Addiction Ontology (see https://addictovocab.org/). Comments and suggestions for improvements are welcome using the Qeios review system.

Definitions imported from the Addiction Ontology (AddictO) are what are known as 'ontological definitions'. See this article in Qeios for an explanation https://www.qeios.com/read/YGIF9B.

Ontological definitions can sometimes be hard to read. In those cases we also include an informal definition.

Definitions also often require elaboration to make it clear how they should be used and what they include. In those cases we include a comment.

Also, definitions sometimes require an explanation as to how they came about to help users understand how they relate to alternative definitions. In those cases we include a curator note. 